\title{
Recurrent Brain Metastases: The Role of Resection of in a Comprehensive Multidisciplinary Treatment Setting
}

Nadine Hessler

University Hospital Cologne: Uniklinik Koln

Stephanie T Jünger

University Hospital Cologne: Uniklinik Koln

Anna-Katharina Meissner

University Hospital Cologne: Uniklinik Koln

Martin kocher

University Hospital Cologne: Uniklinik Koln

Roland H Goldbrunner

University Hospital Cologne: Uniklinik Koln

Stefan Josef Grau ( $\nabla$ stefan.grau@me.com )

University Hospital Cologne https://orcid.org/0000-0002-9742-527X

Research article

Keywords: recurrent brain metastasis, radio-oncological treatment, overall survival

Posted Date: May 18th, 2021

DOl: https://doi.org/10.21203/rs.3.rs-531232/v1

License: (9) (i) This work is licensed under a Creative Commons Attribution 4.0 International License.

Read Full License 


\section{Abstract}

\section{Background}

To evaluate the efficacy of surgical resection for pretreated, recurrent brain metastases (BM) in a comprehensive multidisciplinary treatment setting.

\section{Methods}

In a retrospective single center study, patients were analyzed who had undergone surgical resection of recurrent BM between 2007 and 2019. Intracranial event-free survival (EFS) and overall survival (OS) were evaluated by Kaplan-Maier and Cox regression analysis.

\section{Results}

In total, 107 patients with different primary tumor entities and individual previous treatment for BM were included. Primary tumors comprised non-small cell lung cancer (NSCLC) (37.4\%), breast cancer (19.6\%), melanoma (13.1\%), gastro-intestinal cancer (10.3\%) and other, rare entities (19.6\%). The number of previous treatments of BM ranged from one to four; these comprised: resection only, focal or whole brain radiotherapy, brachytherapy and radiosurgery. BM-related symptoms were present in $73.8 \%$ of the patients. Median pre-operative Karnofsky Performance Score (KPS) was 70\% (range 40-100) which was improved to $80 \%$ (range $0-100$ ) after surgery. The complication rate was $26.2 \%$ and two patients died during the perioperative period. Postoperative local radio-oncologic and/or systemic therapy regimens were applied in 67 (62.6\%) patients. Median postoperative EFS and OS were $7.1(95 \% \mathrm{Cl} 5.8-8.2)$ and 11.1 (95\% Cl 8.4-13.6) months, respectively. The clinical status (postoperative KPS $\geq 70$ (HR $0.2795 \% \mathrm{Cl} 0.16$ $0.46 ; p<0.001)$ remained the only independent factor for survival in multivariate analysis.

\section{Conclusion}

Surgical resection of recurrent BM may improve the clinical status and thus OS but is associated with a high complication rate; therefore, careful patient selection is crucial.

\section{Background}

Due to rising medical standards, multidisciplinary treatment options including novel therapeutic regimens, the number of patients with brain metastases (BM) is increasing [1-4]. Although BM are considered, in principle, a fatal event for oncological patients, treatment paradigms are changing, and affected patients are nowadays frequently treated with repeated non-invasive therapeutic procedures such as radiotherapy and systemic oncological treatments. While the role of neurosurgical resection of primary and symptomatic BM is clearly defined $[5,6]$, the application of surgery for recurrent BM, especially after previous multimodal treatments, remains an individual decision [7], particularly since underlying studies $[8,9]$ are scarce and mostly focus on narrowly defined, rather than heterogeneously pretreated "real-life" patient cohorts. 
In the light of an increasing number of multidisciplinary comprehensive oncological treatmentoptions, including several types of focused radiotherapy and targeted medical treatments with a reported overall survival (OS) benefit, the role of neurosurgery in the context of relapse, especially for symptomatic BM, needs to be clearly defined.

\section{Methods}

\section{Selection of study population}

For this retrospective, monocentric cohort study, we queried our database for patients who had undergone resection of previously treated, recurrent BM in our department between 2007 and 2019. The following parameters were identified: demographic/baseline characteristics (gender, age at time of diagnosis and at time of surgery of the recurrent BM), tumor characteristics (type of primary tumor, local and systemic tumor status, number and location of recurrent BM, time to recurrence since initial cancer diagnosis, time to recurrence since initial diagnosis of BM), therapeutic interventions (previous treatment, types of

adjuvant therapy, number of previous recurrences), clinical status (neurological symptoms, pre- and postoperative Karnofsky-Performance-Scale (KPS)), and outcome measures (surgery-related complications, time to further recurrence after surgery). Data were retrieved from the electronic hospital database and paper charts. The study was approved by the local ethical committee (reference number: 18-089).

\section{Indication for surgery}

Indication for surgery was based on suspected recurrent BM detected by magnetic resonance imaging (MRI) or, if required, amino acid positron emission tomography (PET). All decisions were made within an interdisciplinary institutional tumor board comprising board-certified neurosurgeons, neuro-oncologists, medical oncologists, neuro-radiologists, neuropathologists, and palliative care physicians. In general, criteria for (re-)operation were accessibility and size of the lesion allowing safe resection, good clinical condition, adjuvant treatment options (re-irradiation, chemotherapy, or molecular therapy), necessity for obtaining tissue diagnosis, rapid progression leading to neurological complications, or no remaining treatment options other than surgery. Histopathological diagnosis was made by the local Departments of Neuropathology or Pathology.

\section{Surgical treatment and follow-up}

The extent of resection was assessed by early postoperative MRI performed within 48 hours after surgery and classified as gross total resection when no residual contrast-enhancing tissue was visible on T1weighted imaging. Any residual contrast enhancement was defined as subtotal resection. Clinical and radiological follow-up was performed in three-monthly intervals. Intracranial failure was defined as newly developing contrast enhancement in brain MR imaging. 
Complications were classified according to the Common Terminology Criteria of Adverse Events (CTCAE) by the National Cancer Institute (NCl) $[10,11]$ (see Table 3).

\section{Statistical analysis}

For descriptive statistics, continuous values are given in median and range, ordinal and categorical variables are stated in numbers and percentages. Post-surgical survival time was calculated from the date of surgery to date of death or last follow-up; patients alive at the time of their last follow up were censored. Event-free survival (EFS) was assumed in the case of no intracranial relapse. Predictive variables for both endpoints were identified by univariate and multivariate analysis. For categorical variables, the log-rank test was used to identify covariates with an influence on EFS and OS and visualized in Kaplan-Meier plots. For continuous variables, Hazard ratios were calculated using Cox regression. P-values $<0.05$ were considered statistically significant. Variables with a significant impact were included in a multivariate Cox regression model. All statistical analyses were performed using SPSS Statistics Version 25 (IBM, Armonk, NY, USA).

\section{Results}

\section{Baseline parameters and demographics}

The study included 107 patients with a median age of 61 (range 26 - 83) years at the time of operation. Forty-three patients (40.2\%) were male. Primary tumor entities comprised non-small cell lung cancer (NSCLC) (37.4\%), breast cancer (19.6\%), melanoma (13.1\%), gastro-intestinal tumor (GIT) (10.3\%) and other, rare entities (19.6\%). At the time of BM relapse, extracranial metastases were present in 61 (57.0\%) patients. Detailed demographic and clinical data are displayed in Table 1.

\section{Table 1: Baseline demographic characteristics and parameters}




\begin{tabular}{|c|c|c|c|c|}
\hline Parameter & No. & $\%$ & Median & Range \\
\hline Age at operation & & & 61 & $26-83$ \\
\hline$£ 65$ years & 75 & 70.1 & & \\
\hline$>65$ years & 32 & 29.9 & & \\
\hline \multicolumn{5}{|l|}{ Gender } \\
\hline Male & 43 & 40.2 & & \\
\hline female & 64 & 59.8 & & \\
\hline \multicolumn{5}{|l|}{ Primary tumor } \\
\hline non-small cell lung cancer & 40 & 37.4 & & \\
\hline Breast cancer & 21 & 19.6 & & \\
\hline Melanoma & 14 & 13.1 & & \\
\hline Gastro-intestinal tumor & 11 & 10.3 & & \\
\hline Other & 21 & 19.6 & & \\
\hline \multicolumn{5}{|l|}{ Extracranial disease } \\
\hline Stable & 46 & 43.0 & & \\
\hline Non-stable & 61 & 57.0 & & \\
\hline \multicolumn{5}{|c|}{ Symptoms (multiple references possible) } \\
\hline Cerebellar & 19 & 17.8 & & \\
\hline Cognitive & 11 & 10.3 & & \\
\hline Hemiparesis & 25 & 23.4 & & \\
\hline Seizures & 14 & 13.1 & & \\
\hline Headache & 22 & 20.6 & & \\
\hline Impaired vision & 15 & 14.0 & & \\
\hline Aphasia & 13 & 12.1 & & \\
\hline Others & 18 & 16.8 & & \\
\hline
\end{tabular}

\section{Previous treatment and clinical status at time of recurrence}

Previous cerebral treatment comprised one or more local and/or systemic therapies including surgery, whole brain radiation therapy (WBRT), focal/partial brain radiation therapy (fRT), stereotactic radiosurgery (sRS) and brachytherapy (BT). The number and detailed information on previous treatment modalities were recorded (Table 2). 
Table 2: Pre- and postsurgical treatment, surgery, and complications

\begin{tabular}{|lll|}
\hline Parameter & No. & $\%$ \\
\hline Previous treatment & & \\
Resection & 44 & 41.1 \\
Radiotherapy & 30 & 28.0 \\
Whole brain radiotherapy & 24 & 22.4 \\
Partial brain radiotherapy & 53 & 49.5 \\
Stereotactic radiosurgery & 8 & 7.5 \\
Brachytherapy & & \\
Number of recurrent BM & 80 & 74.8 \\
BM & 19 & 17.8 \\
2-3 BM & 8 & 7.5 \\
$\geq 4$ BM & & \\
& 78 & 72.9 \\
Extent of resection & 29 & 27.1 \\
Gross total & & \\
Subtotal & 40 & 37.4 \\
& 67 & 62.6 \\
Adjuvant local treatment & 5 & 4.7 \\
None & 49 & 45.8 \\
Radiotherapy & 11 & 10.3 \\
Whole brain radiotherapy & 2 & 1.9 \\
Partial brain radiotherapy & 37 & 34.6 \\
Stereotactic radiosurgery & & \\
Combination & & \\
Postsurgical systemic therapy & 37 & 34.6 \\
Cause of death (n= 73) & 12 & 11.2 \\
Neurological & 2 & 1.9 \\
Systemic & 22 & 20.6 \\
Others & & \\
unknown & & \\
\hline
\end{tabular}

At the time of resection, 79 (73.8\%) patients suffered from BM-related symptoms including vertigo, hemiparesis, cognitive impairment, epilepsy, and headache. The median preoperative Karnofsky performance scale (KPS) was 70 (range 40-100).

\section{Surgical treatment, complications, and adjuvant treatment}

At time of surgery $80(74.8 \%)$ patients suffered from a single recurrent BM, 19 patients (17.8\%) from oligo- (2-3) BM and eight patients (7.5\%) from multiple ( $\geq 4)$ BM. Resection of the target lesion was complete (gross total resection) in $78(72.9 \%)$ patients. Surgery was performed in all patients under general anesthesia with the aid of neuro-navigation, ultrasound, and intra-operative monitoring, if required. Surgery improved the Karnofsky performance scale to a median of 80 (0-100). After resection, adjuvant local treatment was administered in 67 patients (62.6\%), comprising WBRT $(n=5)$, fRT $(n=49)$, stereotactic radiosurgery $(n=11)$, or a combination of the latter two $(n=2)$. Medical treatment was initiated or continued in 37 (34.6\%) patients (Table 2). Surgery-related complications occurred in 28 patients (26.2\%) with two patients dying during the acute phase. Details on postsurgical complications and their grading are displayed in detail in Table 3 . 
Table 3: Complications stratified according to CTCAE (Common Terminology Criteria of Adverse Events)

\begin{tabular}{|llc|}
\hline Complication & $\begin{array}{l}\text { CTCAE } \\
\text { grade }\end{array}$ & $\mathbf{n}$ \\
\hline New neurological deficit & 2 & 6 \\
\hline Wound healing disorder & 2 & 5 \\
\hline $\begin{array}{l}\text { Wound healing disorder requiring surgery (revision, external } \\
\text { drain) }\end{array}$ & 3 & 4 \\
\hline CSF disorder requiring surgery & 3 & 3 \\
\hline Postoperative haemorrhage requiring intervention & 4 & 1 \\
\hline Cerebral ischemia & 4 & 1 \\
\hline Cerebral edema & 4 & 1 \\
\hline Pulmonary embolism & 3 & 2 \\
\hline Carotic artery dissection & 4 & 1 \\
\hline Pneumonia, sepsis & 3 & 2 \\
\hline Postoperative death & 5 & 2 \\
\hline
\end{tabular}

Survival

In 51 patients (47.7\%), a cerebral treatment failure was detected, resulting in a median EFS of $7.1(95 \% \mathrm{Cl}$ 5.8-8.2) months. None of the factors analyzed influenced EFS.

At the time of analysis, 73 (68.2\%) patients had died. Median OS time was 11.1 (95\% Cl 8.4-13.6) months. Three patients $(2.8 \%)$ died within the first 30 days after surgery, two from surgical complications. In the remaining cohort, the causes of death were systemic disease progression in 12 patients $(11.2 \%)$, cerebral progression in 37 patients (34.6\%) and other causes in two patients (1.9\%). In the remaining patients, the cause of death was unspecified.

In univariate analysis, a pre- and postoperative KPS $\geq 70(p=0.002$ and $p<0.001)$ and neurological symptoms caused by BM ( $p=0.036)$ were prognostic for survival, while all other parameters (age, primary, number of BM, location, previous treatment, application and type of local treatment, ongoing systemic treatment, extracranial status) showed no significant impact. In multivariate analysis only the postoperative clinical status (HR $0.20795 \% \mathrm{Cl} 0.0816-0.3436 ; p<0.001)$ remained independent.

\section{Discussion}

Due to closer surveillance during follow-up with routine MR imaging, an increasing number of interdisciplinary treatment options, including effective systemic therapies, the number of patients 
diagnosed with recurrent BM is increasing [1-4]. However, the inevitable question of how to treat these patients adequately after cerebral progression still remains unsolved, especially for patients maintaining a good clinical condition over a longer period of time before BM recurrence [4]. Most studies with respect to treatment of recurrent BM focus on a single treatment option such as (re-) radiosurgery or re-irradiation [12].

Surgery is well established as a first-line treatment for larger and symptomatic BM. However, the role of surgery for pretreated, recurrent BM is not yet defined, and only scarce data, originating from the premolecular era, are available. Only a few studies have reported on the feasibility of (re-)surgery in patients with single or multiple recurrent BM $[8,9,13,14]$. They included narrowly defined patient cohorts previously treated by either surgery $[8,13]$ or sRS $[14,15]$, and reported median survival rates after resection of between 7.5 and 11.5 months. With 11.1 months the survival rate in the present study was within the range of the previously reported data. Furthermore, we did not analyze a narrowly defined cohort, but included patients with heterogenous primary tumors as well as a variety of administered prior treatments. The high rate of fatal cerebral progression in this series compared to previous studies may be due to the fact, that besides surgery, most therapeutic options had already been used, leading to a lack of salvage treatment in the case of further cerebral progression. As surgical resection may result in rapid symptom release by reducing the mass effect, the subsequent improvement in the patient's clinical condition, possibly in combination with a re-evaluation of the tumor's molecular status, may represent the major benefit of surgery. Since a fair clinical status is a prerequisite for radio-oncological and a tailored adjuvant treatment, this may positively influence the outcome, as observed before [16]. However, this benefit could not be observed with statistical significance for the patients in the present study.

Probably, the specific condition of this study's population offers an explanation since it comprises patients who had already undergone extensive oncological treatment. Possible subsequent development of resistance may leave few remaining therapeutic approaches.

In cases of extensive pretreatment by radiotherapy, resection might therefore be the only local treatment option left. As the cerebral progression partly reflects treatment failure of previous irradiation, the negligible impact of postoperative radiotherapeutic measures on either EFS or OS in this present study is not surprising.

The major argument for surgery in this patient cohort may be seen in the clinical improvement which is, in line with the current literature, the strongest predictor for further survival after recurrent BM treatment $[4,8$, 15].

As opposed to the clinical improvement mentioned above, the postoperative complication rate was high and included a critical number of life-threatening complications. This is in contrast to other studies reporting on resection in the setting of initial BM diagnosis, where neurosurgery was usually well tolerated and proved to be feasible and safe $[8,13-15,17,18]$. 
The high incidence of complications may be explained by the general condition of oncological patients. The underlying malignancy and/or multiple varied (systemic) pre-treatments would impair wound healing and hemostasis, and increase cardio-pulmonary complications $[4,19]$. Furthermore, patient age was described as independently correlating with clinical outcome, since comorbidities are more common in elderly patients $[4,20,21]$. In this context, the indication for re-resection of BM must be based upon multidisciplinary consent that takes into account the patients' general condition, the possible (and probable) clinical benefit, and the availability of further treatments.

\section{Conclusion}

Surgical resection of recurrent BM may improve patients' clinical status and possibly indirectly prolong survival but carries a high risk for surgery-related complications. Thus, careful patient selection in a multidisciplinary comprehensive treatment setting is mandatory.

\section{Abbreviations}

BM: brain metastases

BT: brachytherapy

CSF: cerebrospinal fluid

CTCAE: Common Terminology Criteria of Adverse Events

CTx: chemotherapy

EFS: event free survival

fRT: fractioned radiotherapy

GIT: gastro-intestinal tumor

HR: Hazard ratio

KPS: Karnofsky performance scale

MRI: Magnetic resonance imaging

NCl: National Cancer Institute

NSCLC: non-small cell lung cancer

OS: overall survival

PET: positron emission tomography 
RCC: renal cell cancer

SCLC: small cell lung cancer

sRS: stereotactic radiosurgery

TT: targeted therapy

WBRT: whole brain radiation therapy

\section{Declarations}

\section{Ethics approval and consent to participate}

The study was an institutional retrospective study approved by the local ethics committees (University of Cologne approval no. 18-089. Due to the mere retrospective nature of the analysis no patient's consent was required.

\section{Consent for publication}

All authors gave their consent for publication.

\section{Availability of data and material}

The datasets used and/or analyzed during the current study are available from the corresponding author on reasonable request.

\section{Competing interests}

All authors declare no conflict of interests.

\section{Funding}

No funding was raised for this study.

\section{Authors' contribution}

Conceptualization: S.G.

Data acquisition: N.H., S.G., S.T.J.

Review of literature: N.H., S.T.J.

Writing - original draft preparation: N.H. S.T.J., S.G.

Writing - review and editing: R.G., A.-K.M., M.K. 
Tables and Figures: N.H.

Supervision: S.T.J., S.G.

\section{Acknowledgement:}

We thank Dr. Avril Arthur-Goettig (www.bioxpress.de) for language editing.

\section{References}

1. Shen CJ, Lim M, Kleinberg LR. Controversies in the Therapy of Brain Metastases: Shifting Paradigms in an Era of Effective Systemic Therapy and Longer-Term Survivorship. Current Treatment Options in Oncology. 2016;17.

2. Steeg PS, Camphausen KA, Smith QR. Brain metastases as preventive and therapeutic targets. Nature Reviews Cancer. 2011.

3. Chamberlain MC, Baik CS, Gadi VK, Bhatia S, Chow LQM. Systemic therapy of brain metastases: Non-small cell lung cancer, breast cancer, and melanoma. Neuro-Oncology. 2017;19:i1-24.

4. Sankey EW, Tsvankin V, Grabowski MM, Nayar G, Batich KA, Risman A, et al. Operative and perioperative considerations in the management of brain metastasis. Cancer Med. 2019;8:6809-31.

5. Kalkanis SN, Kondziolka D, Gaspar LE, Burri SH, Asher AL, Cobbs CS, et al. The role of surgical resection in the management of newly diagnosed brain metastases: A systematic review and evidence-based clinical practice guideline. Journal of Neuro-Oncology. 2010.

6. Olson JJ, Kalkanis SN, Ryken TC. Congress of Neurological Surgeons Systematic Review and Evidence-Based Guidelines for the Treatment of Adults with Metastatic Brain Tumors: Executive Summary. Clin Neurosurg. 2019;84:550-2.

7. Ammirati M, Cobbs CS, Linskey ME, Paleologos NA, Ryken TC, Burri SH, et al. The role of retreatment in the management of recurrent/progressive brain metastases: A systematic review and evidencebased clinical practice guideline. Journal of Neuro-Oncology. 2010.

8. Schackert G, Schmiedel K, Lindner C, Leimert M, Kirsch M. Surgery of recurrent brain metastases: retrospective analysis of 67 patients. Acta Neurochir. 2013;155:1823-32.

9. Kano H, Kondziolka D, Zorro O, Lobato-Polo J, Flickinger JC, Lunsford LD. The results of resection after stereotactic radiosurgery for brain metastases: Clinical article. J Neurosurg. 2009;111:825-31.

10. Atkinson TM, Ryan SJ, Bennett AV, Stover AM, Saracino RM, Rogak LJ, et al. The association between clinician-based common terminology criteria for adverse events (CTCAE) and patientreported outcomes (PRO): a systematic review. Support Care Cancer. 2016;24:3669-76.

11. Kluetz PG, Chingos DT, Basch EM, Mitchell SA. Patient-Reported Outcomes in Cancer Clinical Trials: Measuring Symptomatic Adverse Events With the National Cancer Institute's Patient-Reported Outcomes Version of the Common Terminology Criteria for Adverse Events (PRO-CTCAE). Am Soc Clin Oncol Educ Book. 2016;35:67-73. 
12. Iorio-Morin C, Mercure-Cyr R, Figueiredo G, Touchette CJ, Masson-Côté L, Mathieu D. Repeat stereotactic radiosurgery for the management of locally recurrent brain metastases. J Neurooncol. 2019;145:551-9.

13. Bindal RK, Sawaya R, Leavens ME, Hess KR, Taylor SH. Reoperation for recurrent metastatic brain tumors. Journal of Neurosurgery. 1995;:600-4.

14. Vecil GG, Suki D, Maldaun MVC, Lang FF, SaWaya R. Resection of brain metastases previously treated with stereotactic radiosurgery. J Neurosurg. 2005;102:209-15.

15. Kano H, Kondziolka D, Zorro O, Lobato-Polo J, Flickinger JC, Lunsford LD. The results of resection after stereotactic radiosurgery for brain metastases: Clinical article. JNS. 2009;111:825-31.

16. Schödel P, Jünger ST, Wittersheim M, Reinhardt HC, Schmidt N-O, Goldbrunner R, et al. Surgical resection of symptomatic brain metastases improves the clinical status and facilitates further treatment. Cancer Med. 2020;9:7503-10.

17. Kamp MA, Dibué M, Niemann L, Reichelt DC, Felsberg J, Steiger H-J, et al. Proof of principle: supramarginal resection of cerebral metastases in eloquent brain areas. Acta Neurochir (Wien). 2012;154:1981-6.

18. Kamp MA, Dibué M, Santacroce A, Zella SM, Niemann L, Steiger $\mathrm{H}-\mathrm{J}$, et al. The tumour is not enough or is it? Problems and new concepts in the surgery of cerebral metastases. Ecancermedicalscience. 2013;7:306.

19. Gaspar L, Scott C, Rotman M, Asbell S, Phillips T, Wasserman T, et al. Recursive partitioning analysis (RPA) of prognostic factors in three Radiation Therapy Oncology Group (RTOG) brain metastases trials. Int J Radiat Oncol Biol Phys. 1997;37:745-51.

20. Moazami N, Rice TW, Rybicki LA, Adelstein DJ, Murthy SC, DeCamp MM, et al. Stage III non-small cell lung cancer and metachronous brain metastases. The Journal of Thoracic Cardiovascular Surgery. 2002;124:113-22.

21. Stark AM, Tscheslog H, Buhl R, Held-Feindt J, Mehdorn HM. Surgical treatment for brain metastases: prognostic factors and survival in 177 patients. Neurosurg Rev. 2005;28:115-9.

\section{Figures}




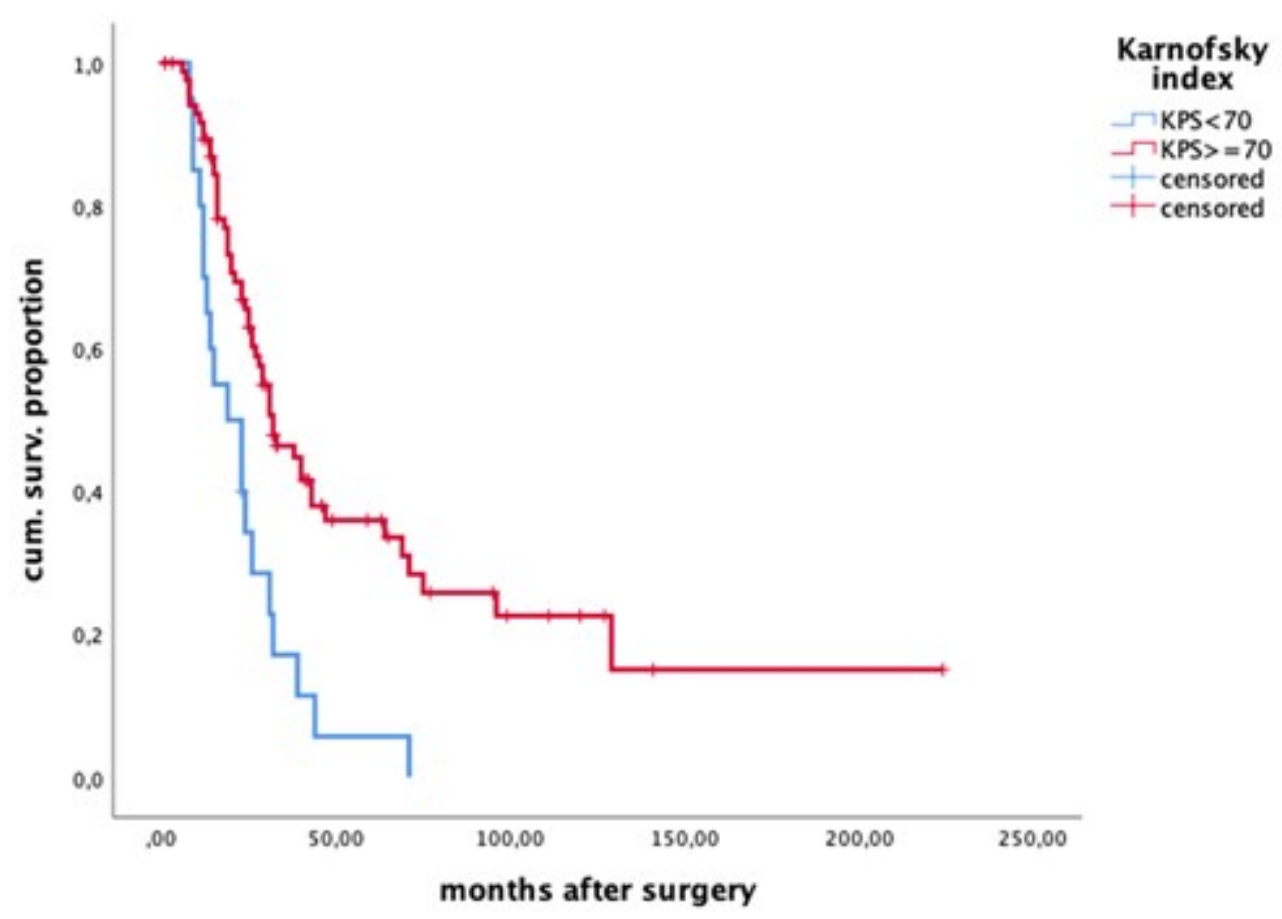

Figure 1

Overall survival (OS), depicting the impact of the clinical status after surgery. Kaplan-Meier plot. 